\title{
An easy and low cost option for economic statistical process control using Excel
}

\author{
PJU van Deventer* $\quad$ ZG Manna ${ }^{\dagger}$
}

Received: 3 December 2008; Revised: 3 March 2009; Accepted: 6 March 2009

\begin{abstract}
In this paper, a user-friendly, Excel program is developed to search for the optimal values of the parameters in minimizing the total cost function in both economic and economic statistical designs of the $\bar{X}$-control chart. Two assumptions are considered in the development and use of the economic or economic statistical models. These assumptions are potentially critical. It is assumed that the time between process shifts can be modelled by means of the exponential distribution. It is further assumed that there is only one assignable cause. Based on these assumptions, economic or economic statistical models are derived using a total cost function per unit time as proposed by a unified approach. In this approach the relationship between the three-control chart parameters as well as the three types of costs are expressed in the total cost function. The optimal parameters are usually obtained by the minimization of the expected total cost per unit time. Nevertheless, few practitioners have tried to optimize the design of their $\bar{X}$-control charts. One reason for this is that the cost models and their associated optimization techniques are often too complex and difficult for practitioners to understand and apply. Therefore, a user-friendly Excel program has been developed in this paper and the numerical examples illustrated are executed on this program. The optimization procedure is easy-to-use, easy-to-understand, and easy-to-access. Moreover, and not least important, it is a low cost option unlike previous approaches which can be found in expensive software packages only. The results and the execution times of all numerical examples show that our optimization procedure using Excel is accurate and efficient.
\end{abstract}

Key words: Statistical design, economic design, economic statistical design, assignable cost, loss cost.

\section{Introduction}

Statistical quality control is a useful and economically important applications of operations research in industry. The purpose of statistical quality control is to ensure, in a cost efficient manner, that the products shipped to customers meet their specifications. One of the basic tools in statistical quality control is the statistical control chart technique

${ }^{*}$ Corresponding author: Department of Statistics and Actuarial Science, University of Stellenbosch, Private Bag X1, Matieland, 7602, South Africa, email: pjuvd@sun.ac.za

${ }^{\dagger}$ Ministry of Finance, Asmara, Eritrea. 
which may be considered a graphical display of statistical hypothesis testing. It was developed in the 1920s by Dr Walter A. Shewhart as a statistical approach to the study of manufacturing process variation for the purpose of improving the economic effectiveness of the process (Shewhart, 1931). The major function of control charting is to detect the occurrence of assignable causes so that the necessary corrective action may be taken before a large number of nonconforming products are manufactured.

Saniga and Shirland (1977) indicated that the $\bar{X}$-chart is used more often than any other control chart when quality is measured on a continuous scale. The effective use of control charts is largely dependent upon their design, that is, the selection of the decision variables such as the sample size, $n$, the sampling period or sampling interval, $h$, and the control limit parameter, $k$, based on some subjective and/or objective criteria.

Due to the economic implications of the control chart design it has received much attention over the years. These economic considerations involve various expenses, such as the costs of sampling and testing, costs associated with investigating out-of-control signals and possibly correcting assignable causes and costs of allowing nonconforming units to reach the customer, etc. Since all these costs are affected by the choice of the three control chart parameters, it is reasonable to consider the design of the $\bar{X}$-control charts from an economic viewpoint.

Consequently, several general methodologies have been developed in order to improve on the design suggested by Shewhart. Economic designs and economic statistical designs are the most important designs that affect the cost and statistical considerations. The concept of an economic design was first introduced by Girschick and Rubin (1952). Although the optimal control rules in their model are too complex to have practical value, their work provided the basis for most cost-based models in control chart designs. Duncan (1956) developed the first economic design model and applied it to an $\bar{X}$-control chart. In the economic design, the objective is to determine the control chart parameters, i.e. the sample size, $n$, sampling interval, $h$, and control limit parameter, $k$, that minimize the expected loss cost accrued by a production process. A considerable amount of research has been done in the economic design of various control charts after Duncan's paper. Lorenzen and Vance (1986) provided a unified approach to the economic design of process control charts. They considered various options regarding continuation of production during search for or repair of the assignable cause. The economic statistical model was first proposed by Saniga (1989). The objective is to minimize the expected total cost per unit time, as in the economic design, subject to constraints on the average run lengths $(A R L)$, or equivalently type I and type II error probabilities or average time-to-signal (ATS).

With regard to the economic and economic statistical designs of the $\bar{X}$-control chart, it can be said that very few practitioners have adopted optimization procedures when designing their $\bar{X}$-control charts. The main reason is that the cost models and their associated optimization techniques are often seen as too complex and difficult for practitioners to apply. Duncan (1956), Gibra (1971), Chiu and Wetherill (1974) and Montgomery (1982) developed the optimization procedures for determining optimal parameters for the $\bar{X}$-control chart. The methodologies described in these papers were difficult to use in practice. The optimization procedure presented by Lorenzen and Vance (1986), for example, employed Newton's method, the golden section search method and the Fibonacci search method. 
This may be one of the reasons for the limited application of these methods. In this paper we propose an alternative optimization procedure, which is a modification to the preceding optimization procedures and we develop a user-friendly Excel program that may be used to calculate the optimal parameter values based on the unified approach of the Lorenzen and Vance model for both economic and economic statistical designs of the $\bar{X}$-control chart. Hypothetical examples are used to show that the proposed optimization procedure works well. Based on this optimization procedure, comprehensive comparisons of the economic and economic statistical designs of the $\bar{X}$-control chart are made. The optimization procedure is easy-to-use, easy-to-understand, and easy-to-access. Moreover, it is a low cost option unlike the previous approaches. The results and the execution times of all numerical examples show that our Excel-implemented optimization procedure is accurate and efficient.

In $\S 2$ two a general review of the approach to the economic statistical design of the $\bar{X}$ control chart is given. This is followed in $\S 3$ by a detailed discussion of the model, while $\S 4$ contains a discussion of the optimization procedure together with the code. The article is concluded in $\S 5$ with two examples together with a detailed discussion of how the results are obtained and interpreted, followed by a brief conclusion $(\S 6)$.

\section{Design of the $\bar{X}$-control chart - A unified approach}

The control chart design has received much attention since the design has behavioural, economic, as well as quality implications (Saniga, 1992). As a result, several general methodologies have been developed to improve on the design suggested by Shewhart. There are two general methods of designing control charts in use today. These methods are the statistical design and the economic design. In statistical design, one considers statistical properties, such as the type I and type II error and the average run length, when selecting the parameters for the control chart (Saniga, 1991). Here the objective is to have control charts signal shifts in the process quickly and accurately, and to keep false signals to a minimum. In the case of the purely statistical design, cost is of no significance. Woodall (1985) addressed the issue of statistical design. In economic design, however, the objective is to determine the control chart parameters that minimize the expected loss cost occurring in a production process.

An alternative to statistical and economic designs has been proposed by Saniga (1989) and is known as the economic statistical design. The economic statistical design is a method in which statistical constraints, such as a minimum value (lower bound) on the in-control $A R L\left(A R L_{L}\right)$ and maximum value (upper bound) on the out-of-control $A R L\left(A R L_{L}\right)$, are placed in the pure economic model so as to yield a design that meets statistical requirements at which the loss cost function is minimized (Montgomery et al., 1995). Economical statistical design was proposed by Saniga (1989) in order to improve both the statistical properties and the economical properties of the resulting control charts (McWilliams, 1994).

Alternatively, the average run length in units of time to a signal (ATS) may be used to replace $A R L$ in the formulation of the design model (Montgomery et al., 1995). Linderman and Love (2000a) showed that on the basis of the selected statistical constraints, control 
charts are designed to have large $A R L_{0}$ or $A T S_{0}$ values when the process is in control, and small $A R L_{1}$ or $A T S_{1}$ values when the process is out of control.

The problem of optimal economic statistical design of control charts may be formulated using $A R L$ and $A T S$ constraints. Let $F$ be the loss cost function for an economic model. In the model for an economic statistical design the objective is to

$$
\begin{array}{ll}
\text { minimize } & F(n, h, k) \\
\text { subject to } & A R L_{0} \geq A R L_{L}, \\
& A R L_{1} \leq A R L_{U},
\end{array}
$$

where $A R L_{L}$ and $A R L_{U}$ denote the desired bounds at the expected shift level. The solution to this model is an improvement to the pure statistical design, because it has the required statistical properties and still minimizes the lost cost function. A solution without the constraints will give the optimal economic design. Montgomery et al. (1995) showed that additional constraints may be added to the design model if sensitivity to shifts that are different from the expected shifts is required.

Economic statistical designs are determined via non-linear constrained optimization techniques. The objective is to minimize the expected total cost per unit time, as in the economic design case, subject to constraints on the type I error rate, power, and ATS (Montgomery et al., 1995). Alternative and additional constraints may be specified depending on the designer's needs. Economic statistical designs are the constrained version of economic designs. If the constraints alone are used in determining design parameters, without considering the cost objectives, they become statistical designs. Zhang and Berardi (1997) showed that economic statistical designs are generally more costly than economic designs due to the additional constraints. However, the tight limits on the statistical properties of the control charts may lead to low process variability that enhances output quality which leads to a reduction in the cost of comebacks and rewards.

\section{The model}

\subsection{Assumptions and notation}

The following assumptions are made for the economic and economic statistical design of control charts:

- The process is subject to a single assignable cause.

- The time between occurrences of the assignable cause is exponential with a mean of $\theta$ occurrences per hour (hence $1 / \theta$ hours is the mean time in the in-control state).

- The process starts in a state of statistical control with mean $\mu_{0}$ and standard deviation $\sigma$.

- The occurrence of the assignable cause results in a shift in the process mean from $\mu_{0}$ to $\mu_{0}+\delta \sigma$, where the shift size $\delta$ is known.

- If a single sample point falls outside the control limits, the process is assumed to be out-of-control and a search for the assignable cause is initiated. 
- The economic and economic statistical designs of control charts assume a renewal reward process. In essence, the corrective actions are assumed to return the process to the initial state of statistical control.

Lorenzen and Vance (1986) established a unified approach to the economic and economic statistical design of the $\bar{X}$-control chart and a unification of notation. The parameters in the formulation of the cost function may be classified into four categories, namely cost and operating parameters, indicator variables and control chart design parameters. The definitions of these parameters are given in the subsections that follow.

\subsection{Cost and operating parameters}

Denote the expected time of occurrence of the assignable cause between two samples, while in control, by $\tau$ and let $\theta$ denote the mean time between occurrences. Furthermore, let $s$ denote the expected number of samples taken while in control, and denote the fixed cost per sample by $a$. Denote the shift size in the mean and the cost per unit sampled by $\delta$ and $b$, respectively. Moreover, let $Y$ and $W$ denote respectively the cost per false alarm and the cost to locate and repair the assignable cost. Also let $C_{0}$ denote the quality cost per hour while producing in control, and denote this cost while producing out of control by $C_{1}$. Denote the time to sample and chart one item by $g$. Furthermore, let $T_{0}$ denote the expected search time when the signal is a false alarm, let $T_{1}$ denote the expected time to discover the assignable cause and let $T_{2}$ denote the expected time to repair the process. Also, denote the total cost per cycle by $C$ and the total cost per time unit by $L$. Moreover, let $A R L_{0}$ denote the average run length while in control and let $A R L_{1}$ denote the average run length while out of control, letting $A R L_{L}$ and $A R L_{U}$ denote the lower bound and upper bound of the average run length while in and out of control, respectively. Lastly, let $A T S$ denote the average time to signal and denote the upper bound of average time-to-signal by $A T S_{U}$.

\subsection{Indicator variables}

The indicator variables

$$
\gamma_{1}= \begin{cases}1, & \text { if production continues during search } \\ 0, & \text { if production ceases during search }\end{cases}
$$

and

$$
\gamma_{2}= \begin{cases}1, & \text { if production continues during repair } \\ 0, & \text { if production ceases during repair, }\end{cases}
$$

are used in the model formulation.

\subsection{Three control chart design parameters}

We denote the sample size by $n$ and the sampling interval by $h$. Finally, the width of the control interval (in terms of the number of standard errors from the mean) is denoted by $k$. 


\subsection{The mathematical model}

The production cycle is defined as the length of time from when the process is started in the in-control state to when it shifts to the out-of-control state, and onwards to where in time the detection and elimination of the assignable cause takes place.

It is known that the expected cycle time is

$$
E(T)=\frac{1}{\theta}+\left(1-\gamma_{1}\right) s \frac{T_{0}}{A R L_{0}}-\tau+n g+h\left(A R L_{1}\right)+T_{1}+T_{2}
$$

(Lorenzen and Vance, 1986). The costs per cycle are incurred by defective production while in-control as well as out-of-control, by false alarms, by locating and repair of the assignable causes, and also by sampling and inspection procedures.

Lorenzen and Vance (1986) showed that the total quality cost per cycle is

$$
\begin{aligned}
E(C)= & \frac{C_{0}}{\theta}+C_{1}\left(-\tau+n g+h\left(A R L_{1}\right)+\gamma_{1} T_{1}+\gamma_{2} T_{2}\right)+\frac{s Y}{A R L_{0}}+W \\
& +(a+b n)\left(\frac{\frac{1}{\theta}-\tau+n g+h\left(A R L_{1}\right)+\gamma_{1} T_{1}+\gamma_{2} T_{2}}{h}\right) .
\end{aligned}
$$

Because the cycle time is variable and is a function of $n, h$ and $k$, the cost function must be expressed per unit of time (e.g. in hours), and not per cycle. Note that since this is a renewal reward process (Ross, 2000), the expected cost per unit of time is found by dividing the total quality cost per cycle, by the expected cycle length, resulting in

$$
\begin{aligned}
E(L)= & \frac{\frac{C_{0}}{\theta}+C_{1}\left(-\tau+n g+h\left(A R L_{1}\right)+\gamma_{1} T_{1}+\gamma_{2} T_{2}\right)+\frac{s Y}{A R L_{0}}+W}{\frac{1}{\theta}+\left(1-\gamma_{1}\right) s \frac{T_{0}}{A R L_{0}}-\tau+n g+h\left(A R L_{1}\right)+T_{1}+T_{2}} \\
& +\frac{a+b n}{h}\left(\frac{\frac{1}{\theta}-\tau+n g+h\left(A R L_{1}\right)+\gamma_{1} T_{1}+\gamma_{2} T_{2}}{\frac{1}{\theta}+\left(1-\gamma_{1}\right) s \frac{T_{0}}{A R L_{0}}-\tau+n g+h\left(A R L_{1}\right)+T_{1}+T_{2}}\right) .
\end{aligned}
$$

\section{The optimization procedure}

As stated above, the function $E(L)$ represents the expected cost per unit of time for the present model and it should be noted that $E(L)$ is a function of the three quality control chart parameters (i.e. the sample size $n$, the sampling period $h$, and the control limit width parameter $k$ ). Note that $A R L_{0}$ and $A R L_{1}$ are functions of $\alpha$ and $1-\beta$, whereas $\alpha$ and $1-\beta$ are, in turn, also functions of $n$ and $k$. As a result, the function $E(L)$ is highly nonlinear in each of the three parameters $n, h$ and $k$.

As mentioned, the algorithm by Lorenzen and Vance (1986) for finding the most economical design is complicated as it consists of Newton's method, the golden section search method and the Fibonacci search method. Montgomery (2001) noted that very few practitioners have implemented economic models for the design of control charts, and one of the reasons for this may be as a result of the complexity of the solution approach. Thus there are at least two reasons for the lack of the practical implementation of this methodology. First, the mathematical models and their associated optimization schemes are relatively complex 
and are often presented in a manner that is difficult for practitioners to understand and to use. A second problem is the difficulty in estimating costs and other model parameters.

Here we present a user-friendly Excel program that may be used to determine an economic or economic statistical design for a $\bar{X}$-control chart. This program uses the model of Lorenzen and Vance, and is configured to be applicable to most actual production situations.

Lorenzen and Vance (1986) showed that the expected loss cost per hour of operation may be expressed as in (3) and may be rewritten as

$$
E(L)=\frac{N U M_{1}}{D E N}+\frac{N U M_{2}}{D E N}
$$

where

$$
\begin{aligned}
& N U M_{1}=\frac{C_{0}}{\theta}+C_{1}\left(-\tau+n g+h\left(A R L_{1}\right)+\gamma_{1} T_{1}+\gamma_{2} T_{2}\right)+\frac{s Y}{A R L_{0}}+W, \\
& N U M_{2}=(a+b n)\left(\frac{\frac{1}{\theta}-\tau+n g+h\left(A R L_{1}\right)+\gamma_{1} T_{1}+\gamma_{2} T_{2}}{h}\right)
\end{aligned}
$$

and

$$
D E N=\frac{1}{\theta}+\left(1-\gamma_{1}\right) s \frac{T_{0}}{A R L_{0}}-\tau+n g+h\left(A R L_{1}\right)+T_{1}+T_{2} .
$$

The expected number of samples, $s$, taken while in control is

$$
\begin{aligned}
s & =\sum_{i=0}^{\infty} i P(\text { assignable cause occurs between the } i \text { th and }(i+1) \text { st sample }) \\
& =\sum_{i=0}^{\infty} i\left(e^{\theta h i}-e^{\theta h(i+1)}\right)=\frac{1}{e^{\theta h}-1}
\end{aligned}
$$

and the average time of occurrence of the assignable cause within the $i$-th and $(i+1)$-st interval is given by

$$
\tau=\frac{\int_{i h}^{(i+1) h} \theta(t-h i) e^{-\theta t} \mathrm{~d} t}{\int_{i h}^{(i+1) h} \theta e^{-\theta t} \mathrm{~d} t}=\frac{1-(1+\theta h) e^{-\theta h}}{\theta\left(1-e^{-\theta h}\right)}=\frac{1}{\theta}-\frac{h}{e^{\theta h}-1}
$$

(Montgomery, 2001). This is an exact solution in contrast to Duncan's approximate approach.

Note that the input parameters are $\theta, \delta, a, b, Y, W, C_{0}, C_{1}, g, T_{0}, T_{1}, T_{2}, \gamma_{1}$ and $\gamma_{2}$ which are therefore entered as fixed values. Note further that $n, h$ and $k$ are input as variables.

The program for which the implementation code may be found in the Appendix, calculates the optimal control limit width parameter $k$ and the sampling interval $h$ for several sample sizes $n$, and displays the corresponding values of the expected cost function $E(L)$. 


\section{$5 \quad$ Illustrative examples}

In this section, the economic optimal design is compared to the economic statistical optimal design by means of examples using the routines as defined in the Appendix.

Based on the unified approach of the cost model developed by Lorenzen and Vance (1986), a more detailed comparison and analysis of the economic and economic statistical designs will be performed in a follow-up article in order to investigate the effects of the input parameters as well as the effect of adding constraints to the statistical performance measurements of the loss function.

\subsection{The optimal economic design}

Torng et al. (1995) provide an application of the single objective design of an $\bar{X}$-control chart based on the approach of Lorenzen and Vance. Suppose that the fixed cost of sampling is R0.50 (i.e. $a=0.50$ ), and the variable cost of sampling is estimated at R0.10 (i.e. $b=0.10$ ). Suppose that it takes approximately three minutes (i.e. $g=0.05$ ) hours to acquire and analyze each observation. The magnitude of the process shifts are assumed to be one standard deviation (i.e. $\delta=1$ ), and process shifts occur according to the exponential distribution with a mean frequency of about one every hundred hours of operation (i.e. $\theta=0.01)$. It takes two hours to investigate an action signal (i.e. $T_{1}=2$ ). The cost of investigating a false alarm is R50 (i.e. $Y=\mathrm{R} 50$ ), and a true action signal costs R25 to investigate (i.e. $W=\mathrm{R} 25$ ). The hourly costs for operating in the in-control state and in the out-of-control state are R10 (i.e. $C_{0}=\mathrm{R} 10$ ) and R100 (i.e. $C_{1}=\mathrm{R} 100$ ), respectively. The process continues operation during the search and repair periods of the assignable cause (i.e. $T_{0}=T_{2}=0$ ).

The Excel implementation searches for the set of design parameters that minimizes the total cost. The optimal control limit $k$ and sampling interval $h$ are computed for several values of $n$. The values of the cost function together with the associated in-control and out-of-control average run lengths are shown in Table 1. This is the same approach used by Alexander et al. (1995), Linderman and Love (2000b), and Montgomery (2001). Using the output, Table 1 reveals that the optimal design has $n=12, k=2.6, h=1.9$ hours, with a minimum cost of approximately R14.84 per hour. The in-control and out-ofcontrol average run lengths for this control chart design are 107.268 and 1.240, respectively. Note that the design at $n=13$ has a minimum cost close to the optimum and also has slightly better statistical properties than the optimal design at $n=12$ in the sense that the probability of a false alarm, $\alpha$, is 0.006934 instead of 0.009322 . Furthermore, the power is improved from 0.806234 to 0.817413 at $n=13$. The improvement in statistical performance leads to a wider control limit parameter, i.e. from 2.6 to 2.7.

In the next example the same economic parameters are used, but we apply statistical constraints in terms of $A R L_{L}, A R L_{U}$ and $A T S$. This illustrates the approach of finding an economic statistical design.

Table 1 shows that the probability that a single point falls outside the limits when the process is in-control, is 0.009322 . That is, even if the process remains in-control, an out-ofcontrol signal will be generated every 107 samples, on average. In other words, the average 


\begin{tabular}{|c|c|c|c|c|c|c|c|c|c|c|}
\hline$n$ & $k$ & $h$ & $\alpha$ & $\beta$ & $(1-\beta)$ & $A R L_{0}$ & $A R L_{1}$ & $A T S_{0}$ & $A T S_{1}$ & $\begin{array}{l}E(L) \\
R / h r\end{array}$ \\
\hline 1 & 2.1 & 0.7 & 0.035729 & 0.863366 & 0.136634 & 27.989 & 7.319 & 19.592 & 5.123 & 19.22 \\
\hline 2 & 2.3 & 0.7 & 0.021448 & 0.812032 & 0.187968 & 46.624 & 5.320 & 32.637 & 3.724 & 17.36 \\
\hline 3 & 2.3 & 0.9 & 0.021448 & 0.714938 & 0.285062 & 46.624 & 3.508 & 41.962 & 3.157 & 16.43 \\
\hline 4 & 2.4 & 0.9 & 0.016395 & 0.655416 & 0.344584 & 60.994 & 2.902 & 54.895 & 2.612 & 15.87 \\
\hline 5 & 2.4 & 1.1 & 0.016395 & 0.565106 & 0.434894 & 60.994 & 2.299 & 67.093 & 2.529 & 15.51 \\
\hline 6 & 2.4 & 1.3 & 0.016395 & 0.480264 & 0.519736 & 60.994 & 1.924 & 79.292 & 2.501 & 15.28 \\
\hline 7 & 2.5 & 1.3 & 0.012419 & 0.442059 & 0.557941 & 80.519 & 1.792 & 104.675 & 2.330 & 15.11 \\
\hline 8 & 2.5 & 1.5 & 0.012419 & 0.371294 & 0.628706 & 80.519 & 1.591 & 120.779 & 2.386 & 14.99 \\
\hline 9 & 2.5 & 1.6 & 0.012419 & 0.308538 & 0.691462 & 80.519 & 1.446 & 128.831 & 2.314 & 14.92 \\
\hline 10 & 2.6 & 1.6 & 0.009322 & 0.286963 & 0.713037 & 107.268 & 1.402 & 171.629 & 2.244 & 14.87 \\
\hline 11 & 2.6 & 1.7 & 0.009322 & 0.236803 & 0.763197 & 107.268 & 1.310 & 182.356 & 2.227 & 14.85 \\
\hline 12 & 2.6 & 1.9 & 0.009322 & 0.193766 & 0.806234 & 107.268 & 1.240 & 203.809 & 2.357 & 14.84 \\
\hline 13 & 2.7 & 1.9 & 0.006934 & 0.182587 & 0.817413 & 144.216 & 1.223 & 274.010 & 2.324 & 14.85 \\
\hline 14 & 2.7 & 2.0 & 0.006934 & 0.148785 & 0.851215 & 144.216 & 1.175 & 288.432 & 2.350 & 14.86 \\
\hline 15 & 2.7 & 2.1 & 0.006934 & 0.120401 & 0.879599 & 144.216 & 1.137 & 302.853 & 2.387 & 14.89 \\
\hline 16 & 2.7 & 2.2 & 0.006934 & 0.096801 & 0.903199 & 144.216 & 1.107 & 317.275 & 2.436 & 14.92 \\
\hline 17 & 2.8 & 2.2 & 0.005110 & 0.092900 & 0.907100 & 195.680 & 1.102 & 430.496 & 2.425 & 14.96 \\
\hline 18 & 2.8 & 2.3 & 0.005110 & 0.074561 & 0.925439 & 195.680 & 1.081 & 450.064 & 2.485 & 15.01 \\
\hline 19 & 2.8 & 2.4 & 0.005110 & 0.059510 & 0.940490 & 195.680 & 1.063 & 469.632 & 2.552 & 15.06 \\
\hline 20 & 2.9 & 2.4 & 0.003732 & 0.057960 & 0.942040 & 267.970 & 1.062 & 643.128 & 2.548 & 15.11 \\
\hline
\end{tabular}

Table 1: Optimal economic design of the $\bar{X}$-control chart.

run length while in-control, $A R L_{0}$, is equal to 107. This large number of false alarms introduces additional variability into the process through over-adjustment and destroys confidence in the control procedure. It is desirable to have this value larger so that false alarms are avoided as far as possible. Therefore, an economic statistical design should be investigated due to the high false alarm rate associated with the economic design.

\subsection{Optimal economic statistical design with $A R L$ constraints}

This example illustrates the economic statistical design of the control chart with $A R L$ constraints, using the same parameter values as in the previous example. The starting points for this example are the arbitrarily specified bounds $A R L_{L}=267$ and $A R L_{U}=40$ for $\delta=1$. The reason for using $A R L$ bounds is to constrain the economic statistical design to an in-control $A R L$ value of at least 267, while keeping the out-of-control $A R L$ at a value of at most 40 .

The first $A R L_{0}$ constraint is equivalent to requiring that $\alpha \leq \frac{1}{267}=0.003745$, and the second $A R L_{1}$ constraint is equivalent to requiring that $1-\beta \geq \frac{1}{40}=0.025$ when a one $\sigma$ shift occurs. Thus, to obtain an economic statistical design, we add two constraints, i.e. $A R L_{0} \geq A R L_{L}$ and $A R L_{1} \leq A R L_{U}$ such that $A R L_{0} \geq 267$ and $A R L_{1} \leq 40$.

The results are shown in Table 2. The optimal design has $n=13, k=2.9, h=1.7$ hours, with a minimum cost of R14.90 per hour. The in-control and out-of-control average run length for this control chart design are 267.970 and 1.316 , respectively compared to 107.268 and 1.240 in the economic design. Note that the designs at $n=12$ and $n=14$ has minimum cost close to the optimum. 


\begin{tabular}{|c|c|c|c|c|c|c|c|c|c|c|}
\hline$n$ & $k$ & $h$ & $\alpha$ & $\beta$ & $(1-\beta)$ & $A R L_{0}$ & $A R L_{1}$ & $A T S_{0}$ & $A T S_{1}$ & $\begin{array}{l}E(L) \\
R / h r\end{array}$ \\
\hline 1 & 2.9 & 0.2 & 0.003732 & 0.971235 & 0.028765 & 267.970 & 34.765 & 53.594 & 6.953 & 21.44 \\
\hline 2 & 2.9 & 0.3 & 0.003732 & 0.931324 & 0.068676 & 267.970 & 14.561 & 80.391 & 4.368 & 18.50 \\
\hline 3 & 2.9 & 0.4 & 0.003732 & 0.878585 & 0.121415 & 267.970 & 8.236 & 107.188 & 3.294 & 17.16 \\
\hline 4 & 2.9 & 0.5 & 0.003732 & 0.815939 & 0.184061 & 267.970 & 5.433 & 133.985 & 2.716 & 16.41 \\
\hline 5 & 2.9 & 0.6 & 0.003732 & 0.746633 & 0.253367 & 267.970 & 3.947 & 160.782 & 2.368 & 15.91 \\
\hline 6 & 2.9 & 0.8 & 0.003732 & 0.673829 & 0.326171 & 267.970 & 3.066 & 214.376 & 2.453 & 15.59 \\
\hline 7 & 2.9 & 0.9 & 0.003732 & 0.600348 & 0.399652 & 267.970 & 2.502 & 241.173 & 2.252 & 15.36 \\
\hline 8 & 2.9 & 1.1 & 0.003732 & 0.528529 & 0.471471 & 267.970 & 2.121 & 294.767 & 2.333 & 15.20 \\
\hline 9 & 2.9 & 1.2 & 0.003732 & 0.460172 & 0.539828 & 267.970 & 1.852 & 321.564 & 2.223 & 15.08 \\
\hline 10 & 2.9 & 1.3 & 0.003732 & 0.396554 & 0.603446 & 267.970 & 1.657 & 348.361 & 2.154 & 15.00 \\
\hline 11 & 2.9 & 1.5 & 0.003732 & 0.338476 & 0.661524 & 267.970 & 1.512 & 401.955 & 2.267 & 14.95 \\
\hline 12 & 2.9 & 1.6 & 0.003732 & 0.286342 & 0.713658 & 267.970 & 1.401 & 428.752 & 2.242 & 14.91 \\
\hline 13 & 2.9 & 1.7 & 0.003732 & 0.240234 & 0.759766 & 267.970 & 1.316 & 455.549 & 2.238 & 14.90 \\
\hline 14 & 2.9 & 1.8 & 0.003732 & 0.199990 & 0.800010 & 267.970 & 1.250 & 482.346 & 2.250 & 14.90 \\
\hline 15 & 2.9 & 1.9 & 0.003732 & 0.165281 & 0.834719 & 267.970 & 1.198 & 509.143 & 2.276 & 14.91 \\
\hline 16 & 2.9 & 2.0 & 0.003732 & 0.135666 & 0.864334 & 267.970 & 1.157 & 535.940 & 2.314 & 14.94 \\
\hline 17 & 2.9 & 2.1 & 0.003732 & 0.110645 & 0.889355 & 267.970 & 1.124 & 562.737 & 2.361 & 14.97 \\
\hline 18 & 2.9 & 2.2 & 0.003732 & 0.089694 & 0.910306 & 267.970 & 1.099 & 589.534 & 2.417 & 15.01 \\
\hline 19 & 2.9 & 2.3 & 0.003732 & 0.072297 & 0.927703 & 267.970 & 1.078 & 616.331 & 2.479 & 15.06 \\
\hline 20 & 2.9 & 2.4 & 0.003732 & 0.057960 & 0.942040 & 267.970 & 1.062 & 643.128 & 2.548 & 15.11 \\
\hline
\end{tabular}

Table 2: Optimal economic statistical designs with $A R L$ constraints.

A comparison between the pure economical design and the economic statistical design of the $\bar{X}$-control chart with an $A R L$ constraint shows that the economic statistical design with $A R L$ constraints have wider control limits and smaller sampling intervals than the economic design. The $A R L_{L}$ constraint of the second example leads to a significant reduction in the frequency of false alarms, while the additional cost incurred by imposing the $A R L_{U}$ and $A R L_{L}$ constraints is minimal. Table 2 shows that it is not expensive to achieve the desired statistical properties. We have calculated the percentage increase in the cost of the economic statistical design over that of the economic design, and the increase in overall expected cost is only $0.41 \%$, i.e. from R14.84 to R14.90, which, in many situations, may be a relatively small price to pay in order to achieve the improved statistical performance of the control charts. The false alarm rate is also reduced from 0.009322 to 0.003732 .

The output of the two examples agree very closely with the results of the two examples in the statistical constrained economic EWMA control chart presented by Torng et al. (1995), but since it is sometimes more appropriate in process monitoring to express shift detection performance in time units, economic statistical designs for the $\bar{X}$-control chart also often incorporate average time-to-signal as the statistical constraint (Montgomery et $a l, 1995)$. The desired $A T S$ bounds are then computed by multiplying the $A R L$ by its corresponding sampling interval. The constraint may be written as

$$
A T S_{1}=\frac{h}{1-\beta}=h \cdot A R L_{1}
$$

where each signal shows an out-of-control situation. 


\subsection{Optimal economic statistical design with ATS constraints}

Here we apply the same input parameters as in the previous two examples, but add the $A T S$ constraint to the pure economic model of the $\bar{X}$-control chart. Suppose the statistical constraint $A T S_{1} \leq 1.90$ is added to the example of Torng et al. (1995).

The optimal economic statistical design of the $\bar{X}$-control chart for the proposed model with ATS bound is shown in Table 3. The table shows that the optimal design has $n=12, k=2.6, h=1.5$ hours, with a minimum cost of R14.90 per hour. The average time-to-signal is 1.861 .

To illustrate the effect of the ATS constraint in the economic statistical design, once more using Excel, we compare it to the pure economic design. Table 3 points out that the economic statistical design has a smaller sampling interval, $(i . e . h=1.5)$ for the economic statistical model with ATS constraint (compared to $h=1.9$ for the pure economic model). The out-of-control $A T S_{1}$ for the economic statistical design is much better than the corresponding $A T S_{1}$ for the pure economic design, i.e. 1.861 against 2.357, resulting in a cost increase of only about $0.37 \%$, i.e. from R14.84 to R14.90. Similar results were reported by Saniga (1989) and Montgomery et al. (1995).

The three examples above indicate that economic statistical designs are generally more expensive than economic designs due to the additional constraints. However, the tighter limits on control chart statistical properties are able to guarantee long-term product or service quality and low process variability. This results directly from the requirement that the economic statistical design assures a satisfactory statistical performance.

\begin{tabular}{|c|c|c|c|c|c|c|c|c|c|c|}
\hline$n$ & $k$ & $h$ & $\alpha$ & $\beta$ & $(1-\beta)$ & $A R L_{0}$ & $A R L_{1}$ & $A T S_{0}$ & $A T S_{1}$ & $\begin{array}{l}E(L) \\
R / h r\end{array}$ \\
\hline 1 & 2.2 & 0.2 & 0.027807 & 0.884243 & 0.115757 & 35.962 & 8.639 & 7.192 & 1.728 & 23.13 \\
\hline 2 & 2.4 & 0.3 & 0.016395 & 0.837813 & 0.162187 & 60.994 & 6.166 & 18.298 & 1.850 & 18.50 \\
\hline 3 & 2.5 & 0.4 & 0.012419 & 0.778730 & 0.221270 & 80.519 & 4.519 & 32.208 & 1.808 & 17.00 \\
\hline 4 & 2.3 & 0.7 & 0.021448 & 0.617903 & 0.382097 & 46.624 & 2.617 & 32.637 & 1.832 & 16.20 \\
\hline 5 & 2.4 & 0.8 & 0.016395 & 0.565106 & 0.434894 & 60.994 & 2.299 & 48.795 & 1.840 & 15.68 \\
\hline 6 & 2.5 & 0.9 & 0.012419 & 0.520142 & 0.479858 & 80.519 & 2.084 & 72.468 & 1.876 & 15.36 \\
\hline 7 & 2.5 & 1.0 & 0.012419 & 0.442059 & 0.557941 & 80.519 & 1.792 & 80.519 & 1.792 & 15.20 \\
\hline 8 & 2.6 & 1.1 & 0.009322 & 0.409657 & 0.590343 & 107.268 & 1.694 & 117.995 & 1.863 & 15.05 \\
\hline 9 & 2.5 & 1.3 & 0.012419 & 0.308538 & 0.691462 & 80.519 & 1.446 & 104.675 & 1.880 & 14.97 \\
\hline 10 & 2.6 & 1.3 & 0.009322 & 0.286963 & 0.713037 & 107.268 & 1.402 & 139.448 & 1.823 & 14.93 \\
\hline 11 & 2.6 & 1.4 & 0.009322 & 0.236803 & 0.763197 & 107.268 & 1.310 & 150.175 & 1.834 & 14.90 \\
\hline 12 & 2.6 & 1.5 & 0.009322 & 0.193766 & 0.806234 & 107.268 & 1.240 & 160.902 & 1.861 & 14.90 \\
\hline 13 & 2.6 & 1.6 & 0.009322 & 0.157316 & 0.842684 & 107.268 & 1.187 & 171.629 & 1.899 & 14.90 \\
\hline 14 & 2.7 & 1.6 & 0.006934 & 0.148785 & 0.851215 & 144.216 & 1.175 & 230.746 & 1.880 & 14.92 \\
\hline 15 & 2.8 & 1.6 & 0.005110 & 0.141639 & 0.858361 & 195.680 & 1.165 & 313.088 & 1.864 & 14.95 \\
\hline 16 & 2.7 & 1.7 & 0.006934 & 0.096801 & 0.903199 & 144.216 & 1.107 & 245.167 & 1.882 & 14.99 \\
\hline 17 & 2.8 & 1.7 & 0.005110 & 0.092900 & 0.907100 & 195.680 & 1.102 & 332.656 & 1.874 & 15.04 \\
\hline 18 & 2.9 & 1.7 & 0.003732 & 0.089694 & 0.910306 & 267.970 & 1.099 & 455.549 & 1.868 & 15.09 \\
\hline 19 & 3.0 & 1.7 & 0.002700 & 0.087089 & 0.912911 & 370.379 & 1.095 & 629.645 & 1.862 & 15.16 \\
\hline 20 & 2.8 & 1.8 & 0.005110 & 0.047249 & 0.952751 & 195.680 & 1.050 & 352.224 & 1.889 & 15.22 \\
\hline
\end{tabular}

Table 3: Optimal economic statistical designs with ATS constraints. 


\section{Conclusion}

This paper shows that an optimization procedure implemented in Excel is easy to use in finding optimal solutions to the designs of both the economic and the economic statistical $\bar{X}$-control charts. It is clear that few practitioners have adopted the economic modeling approach to design their control charts, because the cost models and their associated optimization techniques are often too complex and difficult for practitioners to apply and or the cost of the appropriate software may have a negative effect. However, the numerical examples produced in this paper were executed on an Excel program, and the proposed procedure is easy to use, easy to understand and implies low cost as no expensive software is required. Moreover, the proposed procedure is also able to obtain an exact optimal design rather than the approximate designs as derived by Duncan (1956) and other subsequent researchers. Therefore, this procedure may be used to implement both economic and economic statistical designs of $\bar{X}$-control charts. The results and the execution times of all numerical examples show that our optimization procedure using the Excel program, as outlined is accurate and efficient.

\section{References}

[1] Alexander SM, Dillman MA, Usher JS \& Damodran B, 1995, Economic design of control charts using Taguchi loss function, Computers and Industrial Engineering, 28, pp. 671-679.

[2] Chiu WK \& Wetherill GB, 1974, A simplified scheme for the economic design of $\bar{X}$-charts, Journal of Quality Technology, 6, pp. 63-69.

[3] Duncan AJ, 1956, The economic design of $\bar{X}$-charts used to maintain current control of a process, Journal of the American Statistical Association, 51, pp. 228-242.

[4] Gibra IN, 1971, Economically optimal determination of the parameters of an $\bar{X}$-control chart, Management Science, 17, pp. 635-646.

[5] Girschick MA \& Rubin H, 1952, A Bayes approach to a quality control model, Annals of Mathematical Statistics, 23, pp. 114-125.

[6] Linderman K \& Love TE, 2000a, Economic and economic statistical designs for MEWMA control charts, Journal of Quality Technology, 32, pp. 410-417.

[7] Linderman K \& Love TE, 2000b, Economic and economic statistical designs for MEWMA control charts, Journal of Quality Technology, 32, pp. 457-463.

[8] Lorenzen TJ \& VAnCe LC, 1986, The economic design of control charts: A unified approach, Technometrics, 28, pp. 3-10.

[9] MCWilliams TP, 1989, Economic control chart design and the in-control distribution: A sensitivity study, Journal of Quality Technology, 21, pp. 103-111.

[10] Montgomery DC, 1982 Economical design of an $\bar{X}$-control chart, Journal of Quality Technology, 14, pp. 40-43.

[11] Montgomery DC, 2001, Introduction to statistical quality control, $4^{\text {th }}$ Edition, John Wiley, New York (NY).

[12] Montgomery DC, Torng JC-C, Cochran JK \& Lawrence FP, 1995, Statistically constrained economic design of the EWMA control chart, Journal of Quality Technology, 27, pp. 250-256.

[13] Ross SM, 2000, Introduction to probability models, $7^{\text {th }}$ Edition, Harcourt Science \& Technology Company, San Diego (CA).

[14] Saniga EM \& Shirland LE, 1977, Quality control in practice: A survey, Quality Progress, 10, pp. 30-33.

[15] SANiga EM, 1989, Economic statistical design of control charts with an application to $\bar{X}$ and $R$ charts, Technometrics, 31, pp. 313-320. 
[16] Saniga EM, 1991, Joint statistical design of $\bar{X}$ and $R$ control charts, Journal of Quality Technology, 23, pp. 156-162.

[17] SANiga EM, 1992, Robustness and flexibility of economic statistical designs, pp. 268-273 in LENZ H-J, Wetherill GB \& Wilrich P-Th, Frontiers in statistical quality control, $4^{\text {th }}$ Edition, PhysicaVerlang, Berlin, Germany.

[18] Shewhart WA, 1931, Economic control of quality of manufactured product, D Van Nostrand Company, New York (NY).

[19] Torng JC-C, Cochran JK, Montgomery DC \& Lawrence FP, 1995, Implementing statistically constrained economic EWMA control charts, Journal of Quality Technology, 27, pp. 257-264.

[20] Woodall WH, 1985, The statistical design of quality control charts, The Statistician, 34, pp. 155160.

[21] Woodall WH, 1986, Weaknesses of the economic design of control charts, Technometrics, 28, pp. 408-410.

[22] Zhang G \& Berardi V, 1997, Economic statistical design of $\bar{X}$-control charts for systems with Weibull in-control times, Computers and Industrial Engineering, 32, pp. 575-586.

\section{Appendix}

This appendix contains an illustrative example of the procedure described in the paper, as implemented in Microsoft Excel. The procedure described here is for the example in $\S 4.1 .1$ and produces inter alia Table 1 . Table 1 (in the main text) shows only the basic results, i.e. the optimum result for each value of $n$ ranging over the interval $[1,25]$ in steps of 1 , but some calculation steps have been left out.

It is worthwhile noting that the initial parameter values are in a sense arbitrary, but common sense gives an indication of real world values. The sampling size, $n$, runs through the options of 1 through 25. For each value of $n$, the width of control limits parameter, $k$, typically runs from 2.1 to 3.1 in steps op 0.1. For each combination of the values of the sampling size and the width of control limits parameter, the sampling interval parameter, $h$, runs from 0.1 to 5 in steps of 0.1 time units. This then creates $25 \times 10 \times 50$ rows, each with the expected cost value for that particular row.

The parameters are as defined in the first two rows of the spread-sheet according to the example in $\S 4.1 .1$. The values of $\gamma_{1}$ and $\gamma_{2}$ require some explanation. According to $\S 3.3$ these two parameters are both equal to 1 as the process continues operation during the search and repair periods of the assignable cause.

The spreadsheet calculations start in row 6; therefore the initial references are with respect to that row, except for the fixed values according to the specific problem, as defined in row 2. One has to complete columns $\mathrm{A}, \mathrm{B}$ and $\mathrm{C}$ for $n, k$ and $h$, respectively. Then the remaining entries follow as shown below. Thereafter the remaining calculations are made using the extension facility of Excel. The column definitions are given with comments where deemed necessary. The reader is referred to $\S 3.2$ for clarification of the parameters and formulae. 


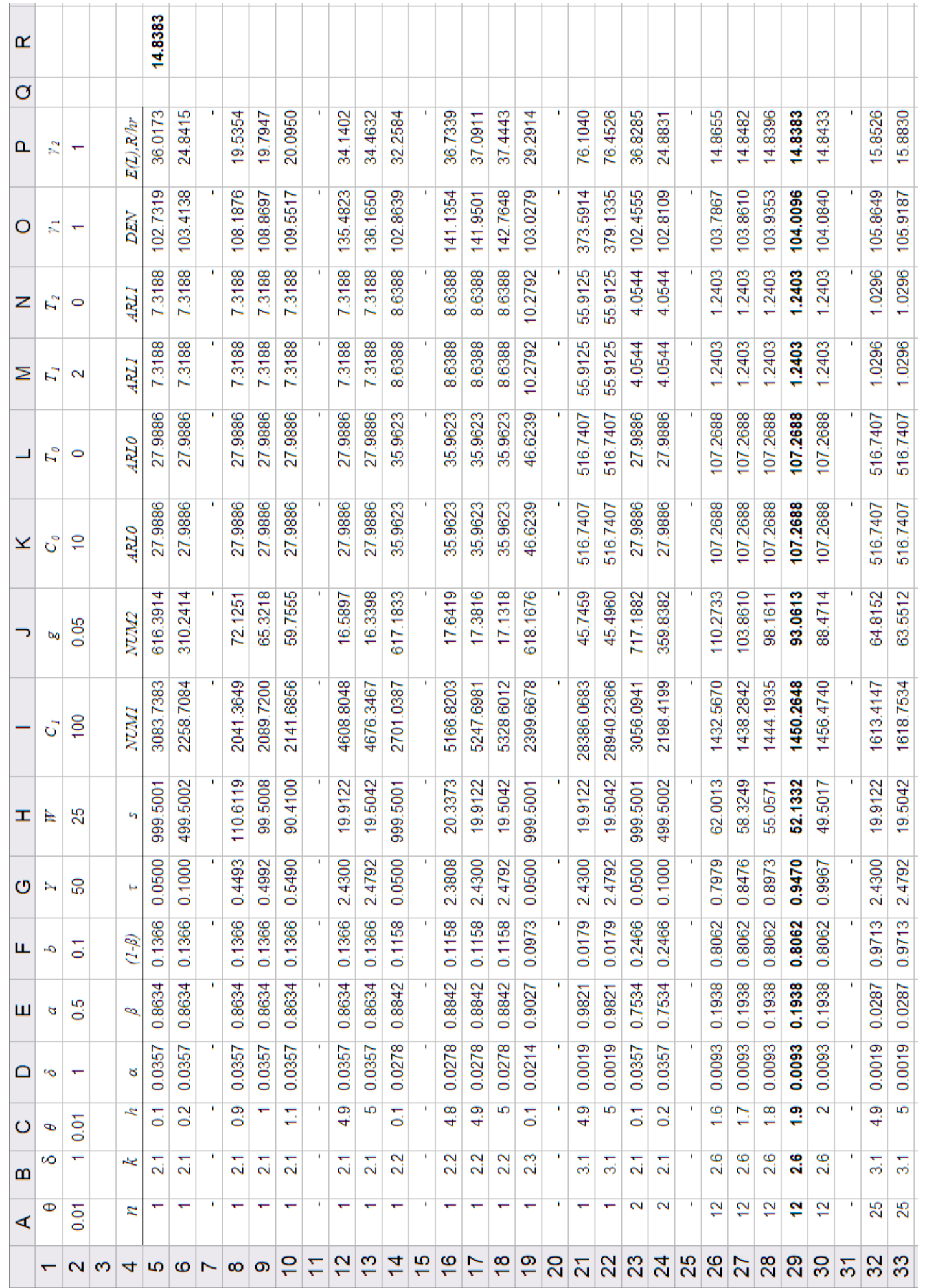

Figure 1: Screen shot of the Excel worksheet in which the model described in this paper has been implemented. 


\section{Column Excel code}

A: $\quad n$ as descibed above.

B: $\quad k$ as descibed above.

C: $\quad h$ as descibed above.

D: $\quad \alpha=2 *$ NORMDIST ( $-\mathrm{B} 6,0,1$, TRUE). The probability of the type I error.

$\mathrm{E}: \quad \beta=\operatorname{NORMDIST}(\mathrm{B} 6-\$ \mathrm{~B} \$ 2 * \operatorname{SQRT}(\mathrm{A} 6), 0,1, \mathrm{TRUE})$

-NORMDIST (-B6-\$B $2 *$ SQRT (A6) , 0,1,TRUE). The probability of the type II error.

F: $\quad 1-\beta=1-$ E6. The power of the underlying hypothesis of an in control system.

G: $\quad \tau=1 / \$ A \$ 2-C 6 /\left((2.7182818)^{\wedge}(\$ A \$ 2 * C 6)-1\right)$. Refer section 4 .

$\mathrm{H}: \quad s=1 /\left((2.7182818)^{\wedge}(\$ \mathrm{~A} \$ 2 * \mathrm{C} 6)-1\right)$. Refer section 4 .

I: $\quad N U M_{1}=\$ I \$ 2 / \$ A \$ 2+\$ G \$ 2 *(-\mathrm{G} 6+\mathrm{A} 6 * \$ \mathrm{H} \$ 2+\mathrm{C} 6 * \mathrm{~N} 6+\$ \mathrm{M} \$ 2 * \$ \mathrm{~K} \$ 2+\$ \mathrm{~N} \$ 2 * \$ \mathrm{~L} \$ 2)$ $+\mathrm{H} 6 * \$ \mathrm{E} \$ 2 / \mathrm{L} 6+\$ \mathrm{~F} \$ 2$. See equation (4) and refer to the explanation for row $\mathrm{P}$.

$\mathrm{J}: \quad \quad N U M_{2}=((\$ C \$ 2 / \$ \mathrm{D} \$ 2 * \mathrm{~A} 6) / \mathrm{C} 6) *(1 / \$ \mathrm{~A} \$ 2-\mathrm{G} 6+\mathrm{A} 6 * \$ \mathrm{H} \$ 2+\mathrm{C} 6 * \mathrm{~N} 6+\$ \mathrm{M} \$ 2 * \$ \mathrm{~K} \$ 2$ $+\$ N \$ 2 * \$ \mathrm{~L} \$ 2)$ See equation (4) and refer to the explanation for row $\mathrm{P}$.

$\mathrm{K}: \quad A R L_{0}=1 / \mathrm{D} 6$.

L: $\quad A R L_{0}=\mathrm{IF}(\mathrm{K} 10>10, \mathrm{~K} 6, " 1 ")$. Put a lower limit of 11 on the average run length if in control. This means that for all practical purposes there is no limit on the $A R L_{0}$ in this example.

$\mathrm{M}: \quad A R L_{1}=1 / \mathrm{F} 6$

$\mathrm{N}: \quad A R L_{1}=\operatorname{IF}(\mathrm{M} 6<=5000, \mathrm{M} 6, " 10000 ")$. Put an upper limit of 5000 on ARL if out of control. This means that for all practical purposes there is no limit on the $A R L_{1}$ in this example.

O: $\quad D E N=1 / \$ \mathrm{~A} \$ 2+(1-\$ \mathrm{M} \$ 2) *(\mathrm{H} 6 * \$ \mathrm{~J} \$ 2) / \mathrm{L} 6-\mathrm{G} 6+\mathrm{A} 6 * \$ \mathrm{H} \$ 2+\mathrm{C} 6 * \mathrm{~N} 6+\$ \mathrm{~K} \$ 2$ $+\$ L \$ 2$. See equation (4) and refer to the explanation for row P. Then use the results of columns I, J and 0 to calculate the cost value for this row with $E(L)=\frac{N U M_{1}}{D E N}+\frac{N U M_{2}}{D E N}$

P: $\quad E(L)=(\mathrm{I} 6+\mathrm{J} 6) / 06$.

R: $\quad M I N=$ MIN (P6:P12505), which was found to be 14.8383 as seen in Table 1 , This is an optimal solution with $n=12, k=2.6$ and $h=1.9$. 
